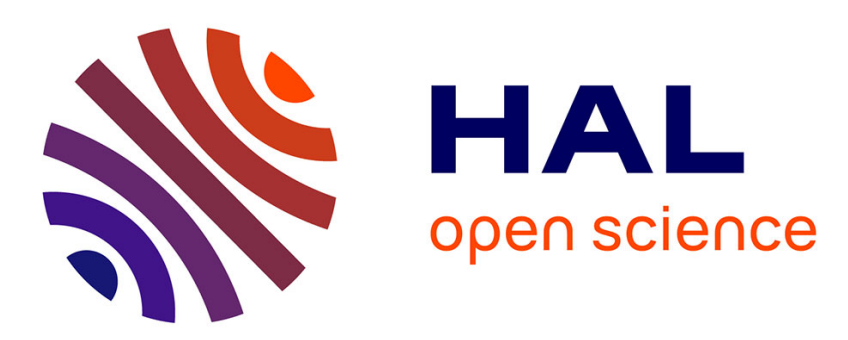

\title{
Active control effectiveness and synchronization of wall turbulence under localized imposed unsteadinesss
}

\author{
Sedat F. Tardu, Olivier Léon Doche
}

\section{To cite this version:}

Sedat F. Tardu, Olivier Léon Doche. Active control effectiveness and synchronization of wall turbulence under localized imposed unsteadinesss. Physics of Fluids, 2007, 19 (108), pp.203-107. 10.1063/1.2793157 . hal-00270777

\section{HAL Id: hal-00270777 \\ https://hal.science/hal-00270777}

Submitted on 5 Feb 2020

HAL is a multi-disciplinary open access archive for the deposit and dissemination of scientific research documents, whether they are published or not. The documents may come from teaching and research institutions in France or abroad, or from public or private research centers.
L'archive ouverte pluridisciplinaire HAL, est destinée au dépôt et à la diffusion de documents scientifiques de niveau recherche, publiés ou non, émanant des établissements d'enseignement et de recherche français ou étrangers, des laboratoires publics ou privés. 


\title{
Active control effectiveness and synchronization of wall turbulence under localized imposed unsteadiness
}

\author{
Sedat F. Tardu ${ }^{\text {a) }}$ and Olivier Doche \\ Laboratoire des Ecoulements Géophysiques et Industriels, Boite Postale 53 X 38041 Grenoble \\ Cédex, France
}

(Received 25 May 2007; accepted 27 August 2007; published online 9 October 2007)

\begin{abstract}
The effect of a spatially localized time-periodic perturbation on the efficiency of suboptimal control of the wall turbulence is analyzed. It is shown that the imposed unsteadiness with a frequency in the median production range doubles the turbulent drag reduction under suboptimal control strategy. It is further observed that the spatially averaged turbulent wall shear is synchronized in time with the imposed perturbation waveform. This is related to the synchronization of the unstable periodic orbits present in the near-wall turbulence in connection with the regeneration cycle of turbulence producing coherent structures. (C) 2007 American Institute of Physics. [DOI: 10.1063/1.2793157]
\end{abstract}

Intensive direct numerical simulation investigations conducted during the past decade have clearly shown that the optimal and suboptimal control of near-wall turbulence are plausible and that appreciable drag reduction can be achieved through either adaptive or nonadaptive schemes. The literature on this topic is vast now and the reader may consult Ref. 1 for some recent ideas and developments. The major shortcoming of these methods is the necessity of a dense distribution of sensors (wall shear stress gauges) and actuators (micro blowing-suction jets) with a mesh size roughly equal to the viscous sublayer thickness to achieve significant drag reduction. Increasing the control mesh size decreases the efficiency of the control scheme. This is not always well understood at first glance. Indeed, the streamwise and spanwise scales of the coherent eddies near the wall are at least an order of magnitude larger than the required control space step. The quasi-streamwise vortices present in the buffer layer are about 300-500 wall units long and are separated by 100 wall units in the spanwise direction. They generate turbulent wall shear by stretching spanwise vorticity zones through ejections and sweeps. However, their regeneration and locations are random in time and space and their capture and subsequent control decision require significantly smaller time and space scales. This poses technical feasibility problems of the suboptimal strategies, despite the important progress achieved in microsmart technologies. Investigations of somewhat simpler large-scale control methods are, therefore, still necessary.

It goes without saying that rendering a process predictable (or deterministic-like) increases the capability of control methods. It is known that any unpredictable process $s[n]$ can be decomposed into $s[n]=s_{\alpha}[n]+s_{\beta}[n]$, where $s_{\alpha}[n]$ is a regular process and $s_{\beta}[n]$ is a predictable process orthogonal to $s_{\alpha}[n]$. This result is known as Wold's decomposition. ${ }^{2}$ In the case of the near-wall turbulence, $s_{\beta}[n]$ may be interpreted as the part due to the coherent structures, while $s_{\alpha}[n]$ is the incoherent part. The aim of a pseudorobust control is then to

${ }^{a}$ Electronic mail: Sedat.Tardu@hmg.inpg.fr intervene locally in space somewhere at the wall to filter $s_{\alpha}[n]$, to accentuate $s_{\beta}[n]$ to control the flow more efficiently at further downstream locations.

The aim of this investigation is to check whether a localized imposed unsteadiness improves the suboptimal control accuracy of the near-wall turbulence or not. We consider a fully developed turbulent channel flow. The flow is locally forced through oscillating blowing by a slot as shown in Fig. 1. The sizes of the slot in wall units in the streamwise $l_{x}^{+}$and spanwise $l_{z}^{+}$directions are shown in this figure. Hereafter, ()$^{+}$ refers to quantities scaled by the inner variables, namely the viscosity $\nu$ and the shear velocity $u_{\tau}=\sqrt{\tau / \rho}$, where $\tau$ is the wall shear stress and $\rho$ is the density. The time-periodic blowing velocity is of the form $\left\langle v_{0}\right\rangle=A \sin (2 \pi f t)$. The imposed frequency is $f^{+}=0.018$ and it is in the median production range of the turbulent kinetic energy spectra $S(f)$, as shown schematically in Fig. 1. The time mean blowing velocity $v_{0}^{+}=A^{+}=0.14$ is significantly small. There is no flow separation downstream the slot under these circumstances. ${ }^{3}$

The effectiveness of control methodology is determined by applying adaptive suboptimal strategy downstream of the oscillating blowing zone as shown in Fig. 1. Contrary to the optimal control whose aim is to laminarize the flow in a given time interval, the suboptimal strategy attempts to decrease at each time step wall shear and the related cost function. The latter is

$$
J(\phi)=\frac{k}{2 \Gamma} \int_{w} \int \phi^{2} d S+\frac{1}{\Gamma} \int_{w} \int \tau d S,
$$

where $\tau$ is the shear at the wall whose area is denoted by $\Gamma$, $\phi$ is the action at the wall in the form of pinpoint blowing/ suction distribution, and $k$ is a constant. The first integral above is clearly the energy expended to achieve the drag reduction. The control problem consists of determining the optimum $\phi$ at each time step. The state equation is the Navier-Stokes equation, 


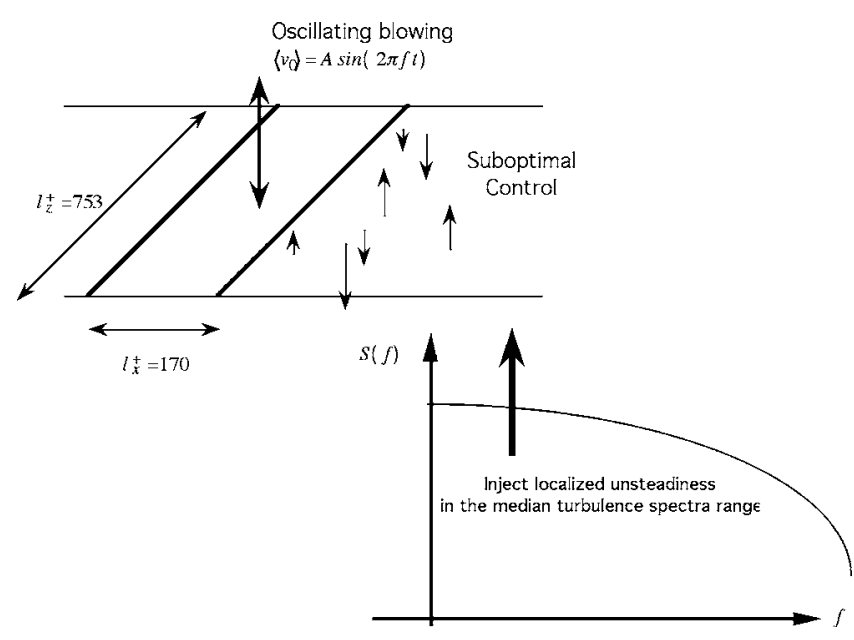

FIG. 1. Wall turbulent dual drag control strategy. The flow is perturbed locally by oscillating blowing through a localized slot. The suboptimal adaptive control is applied downstream (top). The frequency of the localized unsteady perturbation is in the median range of the turbulence spectra (below).

$$
\frac{\partial u_{i}}{\partial t}+\frac{\partial u_{i} u_{j}}{\partial x_{j}}=-\frac{1}{\rho} \frac{\partial P}{\partial x_{i}}+\nu \frac{\partial^{2} u_{j}}{\partial x_{j}^{2}}
$$

where $u_{i}$ and $x_{i}$ are, respectively, the instantaneous local velocity and coordinates, and $P$ denotes the pressure. Mixed notations will be used here for convenience, i.e., the streamwise $\left(x_{1}\right)$, wall normal $\left(x_{2}\right)$, and spanwise $\left(x_{3}\right)$ directions will also be denoted, respectively, by $x, y$, and $z$ together with the corresponding velocity components $u\left(u_{1}\right), v\left(u_{2}\right)$, and $w\left(u_{3}\right)$. Equation (1) is subject to the following boundary conditions at the wall, $x_{2}=y=0$ :

$$
\begin{aligned}
& u_{1}=0, \\
& u_{2}=\phi\left(x_{1}, x_{3}\right), \\
& u_{3}=0 .
\end{aligned}
$$

The sensitivity of the cost function to the actuation modifications $\phi$ is measured through Fréchet derivatives as in classical nonlinear control theory. The variation of a functional $\xi(\phi)$, denoted by $\widetilde{\xi}(\phi, \widetilde{\phi})$, is given by

$$
\tilde{\xi}(\phi, \widetilde{\phi})=\lim _{\varepsilon \rightarrow 0} \frac{\xi(\phi+\varepsilon \widetilde{\phi})-\xi(\phi)}{\varepsilon}=\int_{w} \int \frac{F \xi(\phi)}{F \phi} \widetilde{\phi} d S
$$

where $F$ stands for the Fréchet operator. In practice, the Navier-Stokes equation is discretized in time and space, and the resulting operators are transformed through the Fréchet operator. An adjoint problem is formulated and convenient choice of its boundary conditions allows to relate $D J / D \phi$ to the fluctuating adjoint pressure field at the wall. From $D J / D \phi$, the actuation at the next time step, $n+1$, is computed either by a conjugate gradient method $\phi^{n+1}=\phi^{n}$ $-\alpha(D J / D \phi)^{n}$ or by research of minima algorithm. To be brief, we followed the same procedure as in Bewley et al., ${ }^{4}$ only with some subtle differences.

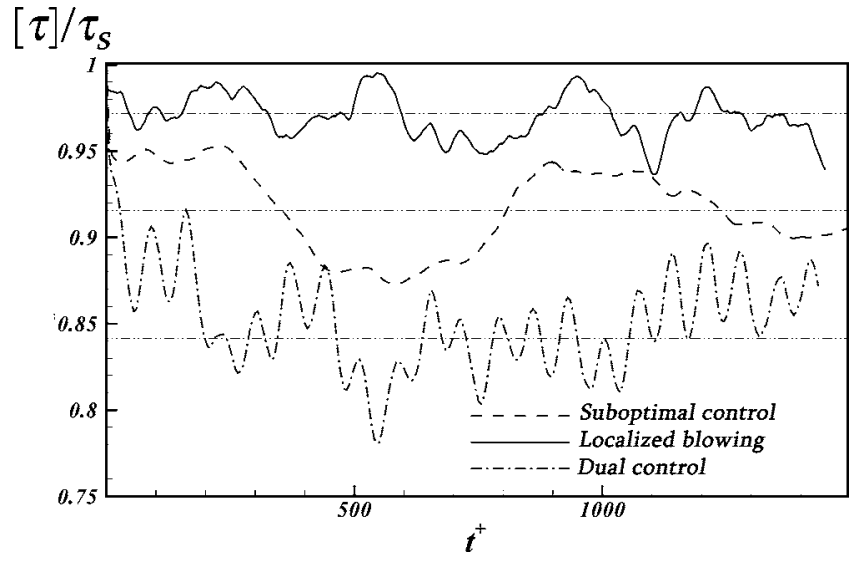

FIG. 2. In situ comparison of the temporal evolution of the spatially averaged wall shear stress under, respectively, the effect of the localized blowing and suboptimal control alone and the dual control that is their combination.

The direct numerical simulations (DNS) are used to model the situation depicted in Fig. 1. The code is of finitedifference-type combined with fractional time procedure. The nonlinear terms are explicitly resolved by an AdamsBash forth scheme. Periodic boundary conditions are used in the homogeneous streamwise and spanwise directions. The size of the computational domain is $(4 \pi h \times 2 h \times 1.33 \pi h)$ in, respectively, the streamwise $x$, wall normal $y$, and spanwise $z$ directions, $h$ standing for the channel half-width. There are $(513 \times 129 \times 129)$ computational modes in $(x, y, z)$. Uniform and stretched coordinates are used in the streamwise, spanwise, and wall normal directions. The first mesh from the wall is at 0.2 wall units. The mesh sizes in the $x$ and $z$ directions are, respectively, 4.5 and $5.5 \quad \ell_{\nu}=\nu / u_{\tau}$. The Reynolds number based on the channel height and the centerline velocity is fixed at $\operatorname{Re}=h U_{c} / \nu=4200$ corresponding to $\operatorname{Re}_{\tau}=h u_{\tau} / \nu=180$. The computational time step is $\Delta t^{+}=\Delta t / \ell_{\nu} u_{\tau}=0.1$.

The important quantity in terms of turbulent drag control is the spatially averaged wall shear stress downstream of the localized unsteadiness. Thus the space-mean shear $[\tau](t)=1 / L_{x}^{+} L_{z}^{+} \int_{0}^{L_{x}^{+}} \int_{0}^{L_{z}^{+}} \tau d x^{+} d z^{+}$is computed and its temporal evolution is analyzed. The zone wherein $[\tau]$ is determined extends up to $L_{x}^{+}=2000$ in the longitudinal direction downstream of the blowing slot. The streamwise averaging extent is twice as large as the correlation length and an order of magnitude greater than the typical length of the coherent near-wall vortices that is roughly 200-300 in wall units. The spanwise extent is that of the computational box, $L_{z}^{+}=753$. Figure 2 shows the temporal evolution of $[\tau]$ for, respectively, under the effect of the localized blowing (LB) and suboptimal control (SC) alone, together with the dual control (DC), which is the combination of LB and SC. The spatially averaged $[\tau]$ is scaled with $\tau_{s}$ of the standard unmanipulated turbulent channel flow. It is seen that the drag downstream of the slot is reduced only by $3 \%$ under the effect of LB alone. LB indeed decreases the drag in an appreciable manner by roughly $30 \%$ in the zone $x^{+}<40$ immediately downstream of the slot, but its effect relaxes rapidly at $x^{+} \approx 400$ at which $\tau$ recovers its standard value $\tau_{s}{ }^{3}$ The suboptimal control re- 


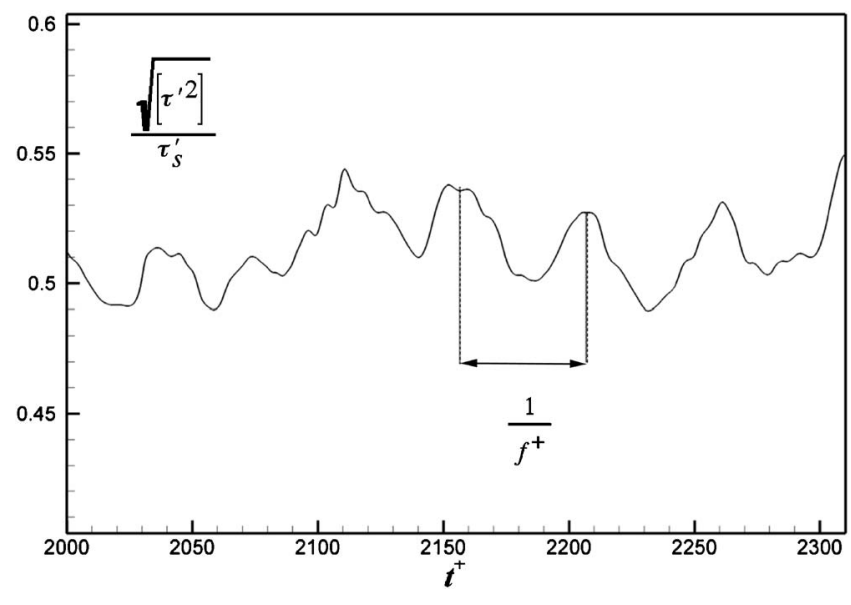

FIG. 3. Temporal evolution of the space-averaged turbulent wall shear stress intensity in the case of dual control. Relatively weak oscillations synchronized with the imposed unsteadiness of frequency $f^{+}$are clearly visible.

sults in $8 \%$ drag reduction. The drag reduction is doubled by dual control and reaches $16 \%$. It has to be emphasized that the unsteady blowing is taken into account in the cost function under the DC strategy. The increase in the efficiency of the suboptimal control in DC is therefore not incidental. The localized unsteadiness increases the suboptimal control effectiveness undeniably.

The second striking feature of the results presented in Fig. 2 is the remarkably smooth temporal evolution of $[\tau]$ that regularly oscillates in the dual control case after the transient period $t^{+}=50$. The frequency of the oscillations in $[\tau]$ is exactly the frequency of the imposed unsteadiness. The amplitude of the oscillations is 5\% and relatively small, but it must be remembered that $[\tau]$ results from averaging in a large domain. Clearly $[\tau]$ is synchronized with the time periodic perturbation velocity $\left\langle v_{0}\right\rangle$ under the effect of dual control.

The turbulence in general and the wall turbulence in particular can be seen as an infinite-dimensional chaotic system. From this particular point of view, the imposed localized unsteadiness not only increases the control efficiency but also leads to a generalized synchronization of its spatiotemporal dynamics. The generalized synchronization (GS) is included in the category of partial synchronization (PS) that refers to the situation wherein some state variables are synchronized but others are not. ${ }^{5}$ The spatially averaged wall shear stress $[\tau]$ is the synchronized flow quantity here. Due to the physics of localized unsteady blowing in the high imposed frequency regime $f^{+} \geqslant 0.01{ }^{3}$, wherein the unsteadiness is confined into the thin low buffer layer $y^{+}<10$, it is unexpected that the spatially averaged flow quantities in the external layer are synchronized. That is, however, unimportant in the context of drag reduction, since the control target here is $[\tau]$, which is clearly synchronized. We observed that the averaged turbulent wall shear stress intensity $\left[\tau^{\prime}\right]^{2}=\left[\tau^{2}\right]$ $-[\tau]^{2}$ exhibits also some periodicity in time and is correlated with the $[\tau]$ modulation. Figure 3 shows the temporal evolution of $\sqrt{\left[\tau^{\prime 2}\right]} / \tau^{\prime}{ }_{s}$ in the case of dual control, where $\tau^{\prime}{ }_{s}$ is the turbulent wall shear stress intensity of the standard channel flow. The turbulent shear activity decreases up to $50 \%$ under essentially the effect of the suboptimal control. That has to be compared with the smaller $16 \%$ decrease in $[\tau]$, resulting in a pronounced disequilibrium of the wall turbulence. There is clearly a dominant frequency equal to that of the blowing velocity modulation in the $\sqrt{\left[\tau^{\prime 2}\right]}$ evolution, as is seen in Fig. 3 . The $\sqrt{\left[\tau^{\prime 2}\right]}$ oscillations are weak but still correlated to $[\tau]$, Therefore, the spatially averaged state variables in the viscous sublayer are functionally related to $[\tau]$ and the process can thus be categorized as GS.

The strategy used here conforms to the original idea of Ott et al. ${ }^{6}$ in the sense that the aimed performance is obtained by making only small time-dependent perturbations to the wall normal velocity system parameter. The periodic perturbation is local in space and the subsequent adaptive suboptimal scheme propagates its effect to a large spatial domain. Both techniques are used in chaos control. ${ }^{7}$ Periodic parametric perturbations applied to low- and highdimensional systems with imposed frequencies corresponding to rational multiples of the frequencies of the periodic orbits (UPO's) result in chaos synchronization. ${ }^{8}$ The UPO's have recently been found in Couette turbulence, and some control strategies inspired from chaos control methodology have been proposed. ${ }^{9}$ The periodic motion embedded in the Couette turbulence is related to the regeneration cycle of the near-wall Reynolds shear stress producing eddies. A directly similar investigation does not exist in the case of fully developed turbulent channel flow. However, it is strongly suspected that UPO's in channel flow are also presumably linked to the genesis of the near-wall coherent vortices. ${ }^{10-12}$ The imposed frequency $f^{+}=0.018$ used here is precisely the regeneration frequency (commonly called ejection frequency $f_{e}^{+}$) of the coherent vortices in the low buffer layer at $y^{+}=20$, where the turbulence production reaches its maximum. ${ }^{13}$ The ejection frequency varies continuously from $f_{e \min }^{+} \approx 0.001$ at the wall to $f_{e \max }^{+} \approx 0.03$ in the loglayer. Further computations we conducted have indeed shown that PS no longer occurs for $f^{+} \geqslant f_{e \text { max }}^{+}$, strengthening the arguments presented here.

It has to be emphasized that the imposed perturbation is spatially local here, and the results are therefore more attractive from a feasibility point of view. The forcing is applied through boundary conditions contrarily to Guan et al. ${ }^{14}$ wherein the streamwise velocity component is unidirectionally coupled with a target state in the whole flow domain. The adaptive suboptimal control applied downstream of the localized perturbation sorts out the UPO and leads to partial synchronization.

In conclusion, we have shown that adding an external frequency to the wall turbulence in the range of the regeneration cycle spectrum increases the effectiveness of the suboptimal control of the turbulent drag. The second effect of the imposed unsteadiness is the coupling with preexisting UPO's leading to a controlled wall shear stress that smoothly oscillates with appreciably less turbulent activity. The present strategy may be applied to achieve active control with significantly less density of the wall controllers using several local oscillators recovering the entire broadband spectra of the wall turbulence in the production range. 
${ }^{1}$ T. Bewley, P. Moin, and R. Temam, "DNS-based predictive control of turbulence: An optimal benchmark for feedback algorithms," J. Fluid Mech. 447, 179 (2001).

2 A. Papoulis, "Predictable processes and Wold's decomposition: A review," IEEE Trans. Acoust., Speech, Signal Process. 33, 933 (1985).

${ }^{3}$ S. Tardu, "Active control of near wall turbulence by oscillating blowing," J. Fluid Mech. 43, 217 (2001).

${ }^{4}$ T. Bewley, H. Choi, R. Temam, and P. Moin, "Optimal Feedback Control of Turbulent Channel Flow," Annual Research Briefs (Center of Turbulence Research, Stanford, 1993), pp. 3-14.

${ }^{5}$ S. Boccaletti, J. Kurths, G. Osipov, D. L. Valladeres, and C. S. Zhou, "The synchronization of chaotic systems" Phys. Rep. 366, 1 (2002).

${ }^{6}$ E. Ott, C. Grebogi, and Y. A. Yorke, "Controlling chaos," Phys. Rev. Lett. 64, 1196 (1990).

${ }^{7}$ S. Boccaletti, C. Grebogi, Y. C. Lai, H. Mancini, and D. Maza, "The control of chaos: Theory and applications," Phys. Rep. 329, 103 (2000).

${ }^{8}$ K. A. Mirus and J. C. Sprott, "Controlling chaos in low and high dimen- sional systems with periodic perturbations," Phys. Rev. E 59, 5313 (1999).

${ }^{9}$ G. Kawahara, S. Kida, and L. van Veen, "Unstable periodic motion in turbulent flows," Nonlinear Processes Geophys. 13, 499 (2006).

${ }^{10}$ P. Holmes, J. L. Lumley, and G. Berkooz, Turbulence, Coherent Structures, Dynamical Systems and Symmetry (Cambridge University Press, Cambridge, 1996)

${ }^{11}$ J. M. Hamilton, J. Kim, and F. Waleffe, "Regeneration mechanisms of near wall turbulence structures," J. Fluid Mech. 287, 317 (1995).

${ }^{12} \mathrm{~F}$. Waleffe, "Homotopy of exact coherent structures in plane shear flows," Phys. Fluids 15, 1517 (2003).

${ }^{13} \mathrm{~S}$. Tardu, "Characteristics of single and clusters of bursting events in the inner region of a turbulent channel flow. Part 2: Level crossing events," Exp. Fluids 33, 640, 2002.

${ }^{14}$ S. Guan, G. W. Wei, and C. H. Lai, "Controllability of flow turbulence," Phys. Rev. E 69, 066214 (2004). 